\section{CONTROL OF SATELLITES WITH ONBOARD ROBOTIC MANIPULATORS}

RoBERT BuCKELEW, ETHAN CATALANELLO, ANNALISA SCACCHIOLI (FACULTY ADVISOR)

\section{* ABSTRACT}

Free-floating satellites with onboard robotic manipulators are subjected to widely varying loads resulting from the motion of the robotic manipulators. As there are no fixed supports in space, these loads will cause the satellite to move. By modelling the motion of the onboard robotic arms, determining the necessary reaction loads (which must be supplied by the satellite to keep the arm fixed), and simulating the resulting satellite dynamics, we designed a model of a satellite-arm system. We found that a Proportional-Integral-Derivative (PID) control scheme, with disturbance-estimating capabilities, was effective in maintaining satellite position and orientation during the operation of the onboard robotic manipulator. The MATLAB-based Simulink modeling environment was used to perform the simulations of satellite dynamics and control.

\section{INTRODUCTION}

The operational success of several fields, including communication technology and space exploration, is dependent on a functional space satellite infrastructure. An advanced satellite network, such as a constellation of communications satellites, will require routine maintenance to maximize its functional lifetime ${ }^{[1]}$. The Defense Advanced Research Projects Agency (DARPA) has proposed technology to perform robotic servicing of on-orbit satellites $^{[2]}$. The project is titled Robotic Servicing of Geosynchronous Satellites (RSGS) and involves satellite- mounted robotic arms which can perform operations on other satellites. Potential missions for the "nurse" satellite include inspection, installation, and repair of on-orbit satellites as well as their relocation to new orbits.

In general, the motion of robotic manipulators and the body to which they are mounted are coupled-that is, the motion of one tends to influence the motion of the other. The magnitude of this effect depends on the inertial properties of the bodies and the interaction forces involved. In a microgravity environment such as Low Earth Orbit (LEO), no dissipative reaction forces exist unlike those present in operations performed on and near the Earth's surface. Therefore, the motion of satellite-mounted robotic manipulators will have significant effects on satellite attitude (the direction the satellite is pointing ${ }^{[3]}$.

It is desirable to reduce the effects of robotic manipulator disturbances on satellite position and attitude using an attitude control system (ACS). An ACS measures satellite position and orientation with respect to reference values. It uses mechanical actuators (e.g., reaction wheels, gas jet thrusters) to reject disturbances. In this project, we model the dynamics of the FREND Mark II robotic arm and simulate the motion of a base satellite subjected to disturbances from the robotic arm motion. Additionally, we designed a control scheme using a basic PID (Proportional-Integral-Derivative) controller. PID controllers feature three gains-proportional, integral, and derivative-which determine the control input signal based on the magnitude, time integration, and time rate of change of the error signal respectively. The goal is to reduce the perturbations arising from operation of the robotic arm, resulting in a satellite that maintains its position and attitude. While extensive work has been performed in this area utilizing complex, nonlinear controls, we wanted to evaluate the effectiveness of a much simpler, linear control scheme, as it was assumed the perturbations arising from the robotic arm would be very small. We believed that these small perturbations would be well approximated by linear control theory, and thus that linear controllers would be sufficient at stabilizing the system. This belief/idea, combined with the 
development of the modular simulation for control design, serves as the novel contribution of our project. We have created an environment that allows for control refinement without requiring rigorous mathematical proofs.

\section{Methodology}

The robotic arm proposed by DARPA for the Robotic Servicing of Geosynchronous Satellites (RSGS) program is the FREND Mark II (hereafter referred to as the FREND), a 7-joint 3D manipulator which will be responsible for performing operations on the "patient" satellite. An illustration of the FREND arm is shown in FIGURE 1 .

The Robotics, Vision, and Control Toolbox, a third-party MATLAB-based toolbox for robotics applications (hereafter referred to as "the Toolbox") was used to develop a model of the FREND and to perform dynamics calculations. The results of these dynamics calculations, based on the FREND model, were used as inputs to the satellite dynamics simulation. These results effectively modeled the disturbances to the satellite arising from the motion of the robotic arm.

\section{FREND Arm Modelling}

The Toolbox is capable of modelling and performing calculations on any robotic arm so long as the arm's relevant parameters are provided. The relevant parameters are inertial parameters, including the mass and inertia matrix and Denavit-Hartenberg parameters (which describe arm geometry). The Denavit-Hartenberg parameters used in the FREND model were defined according to the definitions shown in FIGURE 2 and measurements taken from FIGURE 3. The Denavit-Hartenberg parameters are shown in SUPPLEMENTAL TABLE S1.

IT IS DESIRABLE TO REDUCE THE EFFECTS OF ROBOTIC MANIPULATOR DISTURBANCES ON SATELLITE POSITION AND ATTITUDE USING AN ATTITUDE CONTROL SYSTEM (ACS).

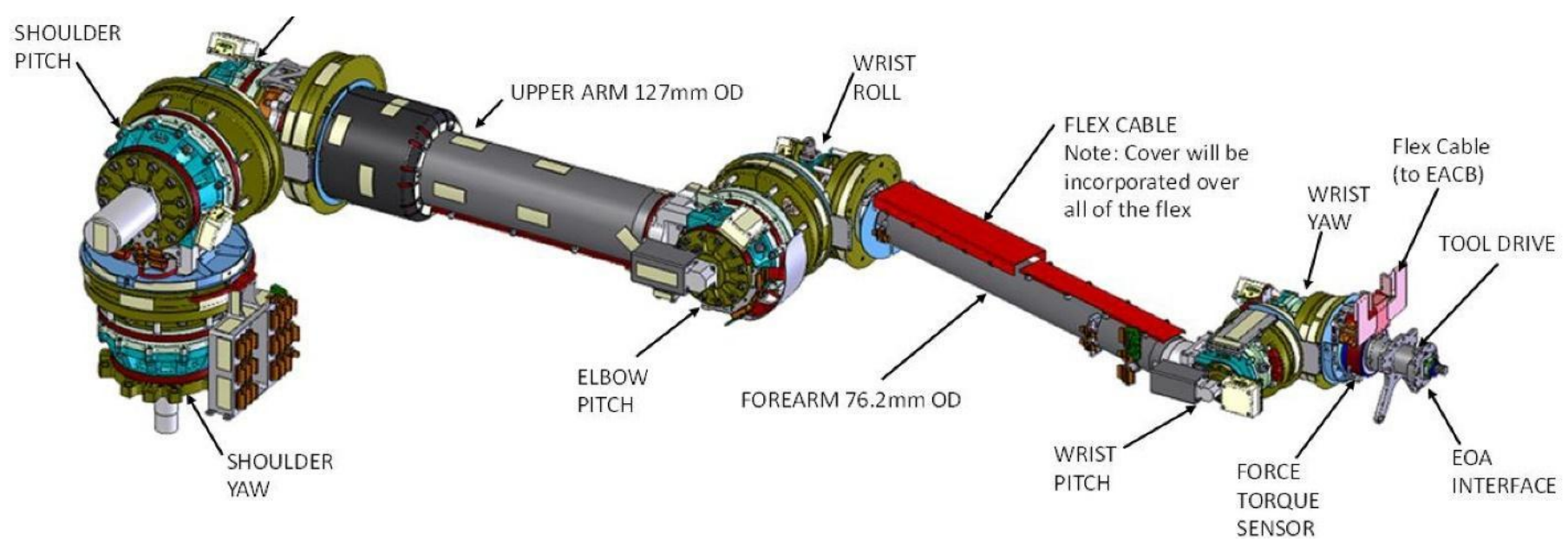

FIGURE 1: DARPA rendering of the FREND Mark // arm ${ }^{[4]}$ 


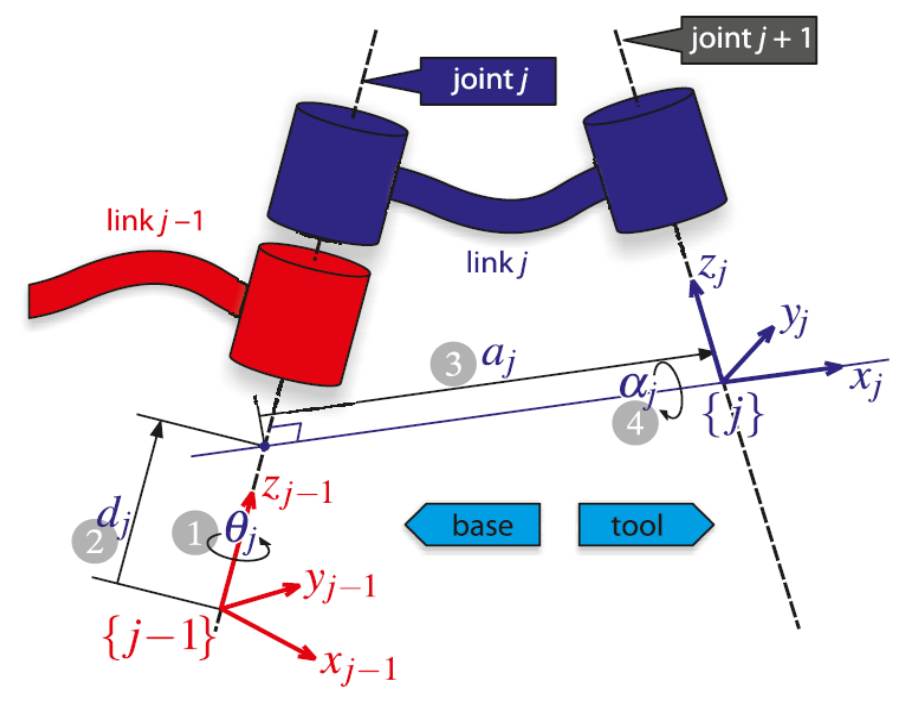

ABOVE:

FIGURE 2: Graphic definition of

Denavit-Hartenberg parameters ${ }^{[5]}$

RIGHT:

FIGURE 3: Schematic of the FREND Mark II showing the relative orientations of link reference frames ${ }^{[4]}$

None of the required parameters for the FREND were publicly accessible, so the measurements were approximated using pixel measurements from schematics and images that were publicly available. Knowing this information, an image editing software can be used to analyze the schematic in FIGURE 3 in order to define a pixel scale which relates real-world dimensions to pixel dimensions. The geometry of the arm was then calculated from the schematic in FIGURE 3 and used to compute the inertia tensor. Each link is assumed to be a cylinder of solid aluminum having a density of $2700 \frac{\mathrm{kg}}{\mathrm{m}^{3}}$ and a center of mass located at the geometric center. This assumption of material composition represents a "worst case scenario" as the actual arm is not solid aluminum ${ }^{[4]}$. The computed dynamic parameters are shown in SUPPLEMENTAL TABLES2 and then inertia matrices are shown in SUPPLEMENTAL TABLE S3.

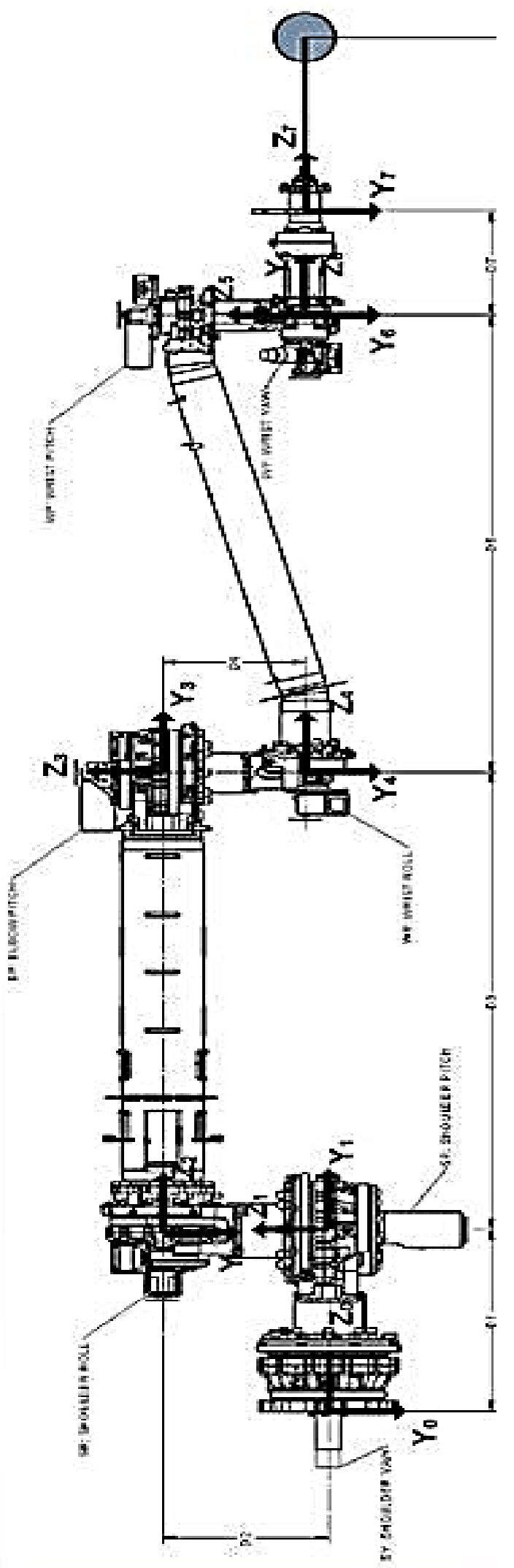


The Toolbox features a function that can numerically evaluate the robot dynamics equation (EOUATION (1)). This is a system of coupled differential equations that relates the joints' angular kinematics $(q, \dot{q}, \ddot{q})$ of the robotic arm joints to the joint torques $(Q)$ and other external loads (end effector wrench $W$ and gravity torque $G(q)^{[a]}$ ) acting on the arm. Solving these differential equations yields the joint torques and the reaction loads at the base of the arm.

$$
Q(q, \dot{q}, \ddot{q})=M(q) \ddot{q}+C(q, \dot{q}) \dot{q}+F(\dot{q})+G(q)+J(q)^{T} W
$$

The parameters of EQUATION (1) are defined in TABLE 1 below.

\begin{tabular}{cl}
\hline$q, \dot{q}, \ddot{q}$ & JOINT ANGULAR POSITION, VELOCITY, and ACCELERATION respectively \\
\hline$Q$ & VECTOR OF GENERALIZED JOINT TORQUES - torques required at each joint to achieve the motion described by $(q, \dot{q}, \ddot{q})$ \\
\hline$M(q)$ & JOINT-SPACE INERTIA MATRIX - describes the moment of inertia of each link in matrix form \\
\hline$C(q, \dot{q})$ & CORIOLIS AND CENTRIPETAL COUPLING MATRIX - describes centripetal forces due to rotation of the links \\
\hline$F(\dot{q})$ & FRICTION MATRIX - describes torques from friction present in the joints \\
\hline$G(q)$ & GRAVITY LOAD - describes torques originating from gravity acting on the links \\
\hline$J(q)$ & MANIPULATOR JACOBIAN MATRIX - describes the sensitivity of the end effector to the motion of each joint \\
\hline$W$ & END EFFECTOR WRENCH - describes the load on the end effector \\
\hline
\end{tabular}

TABLE 1: Definition of terms in EQUATION (1)

The joint torques $Q(q, \dot{q}, \ddot{q})$ obtained through the solution of the differential equation can be utilized to solve for the reaction forces as well as the moments the arm will induce on the satellite. Thus, these loads will be utilized in the simulation as the external disturbance the satellite is subjected to.

\section{Satellite Modelling}

To model the effects of robotic arm motion on satellite position and attitude, we utilized the Aerospace Blockset's "6DOF" (six degree-of-freedom) block in the MATLAB-based Simulink modeling environment. This block implements the equations of motion described in EQUATIONs (2) and (3), which are the differential equations describing translational motion and angular motion, respectively. The parameters of these equations are defined explicitly in SUPPLEMENTAL TABLE S5.

$$
\begin{gathered}
\ddot{x}=\frac{\sum_{j=1}^{N} F_{j}(t)}{m} \\
\ddot{\theta}(t)=I^{-1}\left(\sum_{j=1}^{N}\left(M(t)+r_{j} \times F_{j}(t)\right)-\dot{\theta}(t) \times(I \dot{\theta}(t))\right)
\end{gathered}
$$

\footnotetext{
${ }^{[a]}$ Gravity was neglected in our simulation because the satellite is in space.
} 


\begin{tabular}{cl}
\hline$x(t)$ & VECTOR OF SATELLITE POSITIONAL COORDINATES \\
\hline$\theta(t)$ & VECTOR OF SATELLITE ANGULAR POSITION COORDINATES \\
\hline$F_{j}(t)$ & VECTOR OF TRANSLATIONAL FORCE CAUSED BY ROBOTIC ARM $j$ \\
\hline$M_{j}(t)$ & VECTOR OF MOMENTS CAUSED BY ROBOTIC ARM $j$ \\
\hline$m$ & SATELLITE MASS \\
\hline$I$ & SATELLITE INERTIA TENSOR \\
\hline$r_{j}$ & VECTOR DEFINING THE BASE OF ARM $j$ RELATIVE TO THE SATELLITE COM \\
\hline
\end{tabular}

TABLE 2: Definition of terms in Equations (2) and (3)

Since there is no satellite design specified for the RSGS program, we assumed a generic small satellite configuration. We take the satellite to be a rectangular prism of $2 m \times 1 m \times 1 m$ with two thin rectangular solar arrays fixed at opposing ends $3 m \times 1.5 m \times .0055 m$ located $.5 m$ from the satellite body. The density of the satellite was assumed to be $200 \frac{\mathrm{kg}}{\mathrm{m}^{3}}$, which is an approximate mass density for spacecraft of this size ${ }^{[9]}$ and the solar panels were assumed to be identical to those manufactured by Spectrolab, a manufacturer of satellites for use in space, and have a planar density of $2.06 \frac{\mathrm{kg}}{\mathrm{m}^{2}}{ }^{[10]}$. This means that the entire satellite has a mass of $\mathrm{m}=411.9305362785 \mathrm{~kg}$, and the inertia tensor is shown below:

$$
I=(303.02687565826000170 .14297228667000199 .55068127826) \mathrm{kg} \cdot \mathrm{m}^{2}
$$

\section{Controller Modelling}

Recall that our objective is to maintain the translational position as well as the angular position (or attitude) of the satellite throughout operation of the on-board robotic arm. To accomplish satellite position and attitude control, PID controllers, which represents a basic function for the control of linear systems, were implemented for each of the six degrees of freedom, namely the three translational coordinates $(x, y, z)$ of satellite position and the three Euler angles $(\phi, \theta, \psi)$ defining satellite roll, pitch, and yaw, respectively. The optimal values of the PID controller gains, $K_{P}, K_{I}$, and $K_{D}$ are determined automatically using the MATLAB PID Tuner tool. The optimal gains for the RSGS model satellite are shown in SUPPLEMENTAL TABLE S4.

\section{RESULTS}

As a result of this project, we have developed a multiple component model in which a user can define a robotic arm configuration and trajectory as well as the inertial parameters of a satellite. The user can then simulate the motion response of the satellite to disturbances arising from motion of the arm and employ a predictive control scheme to maintain a reference satellite position and attitude. A simplified Simulink block diagram demonstrating the fundamental logic behind the model is shown in FIGURE 4. As shown in the figure, the controller (which is a PID controller in this case) receives the translational position and angular position offset as well as an estimation of the incoming arm loads which it then uses to calculate the necessary control force/torque. 


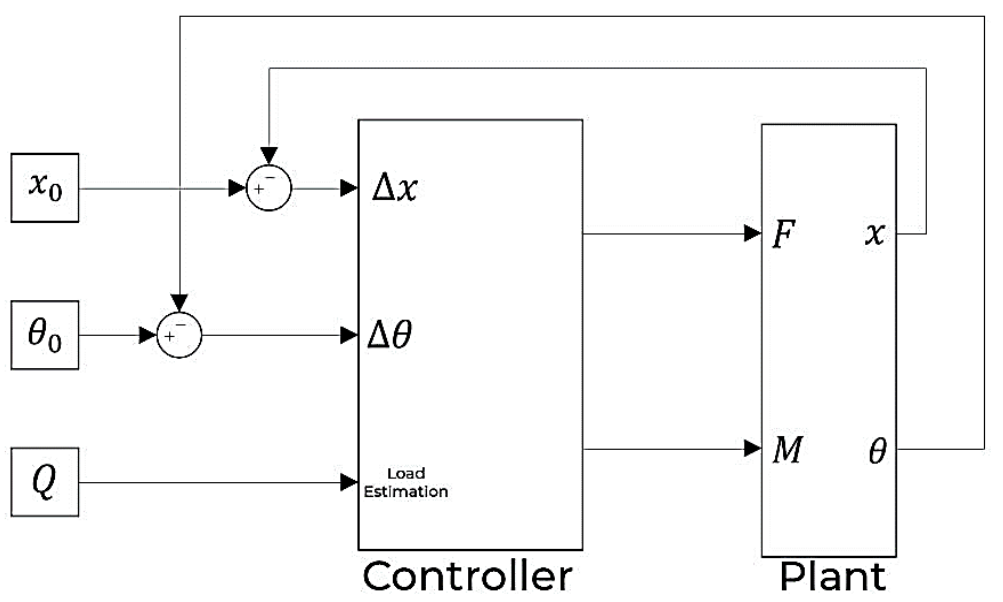

LEFT:

FIGURE 4: Simplified block diagram describing the logical relations between the disturbance load, satellite, and controller

BELOW:

FIGURE 5: Disturbance forces and moments acting on the satellite throughout the arm's trajectory

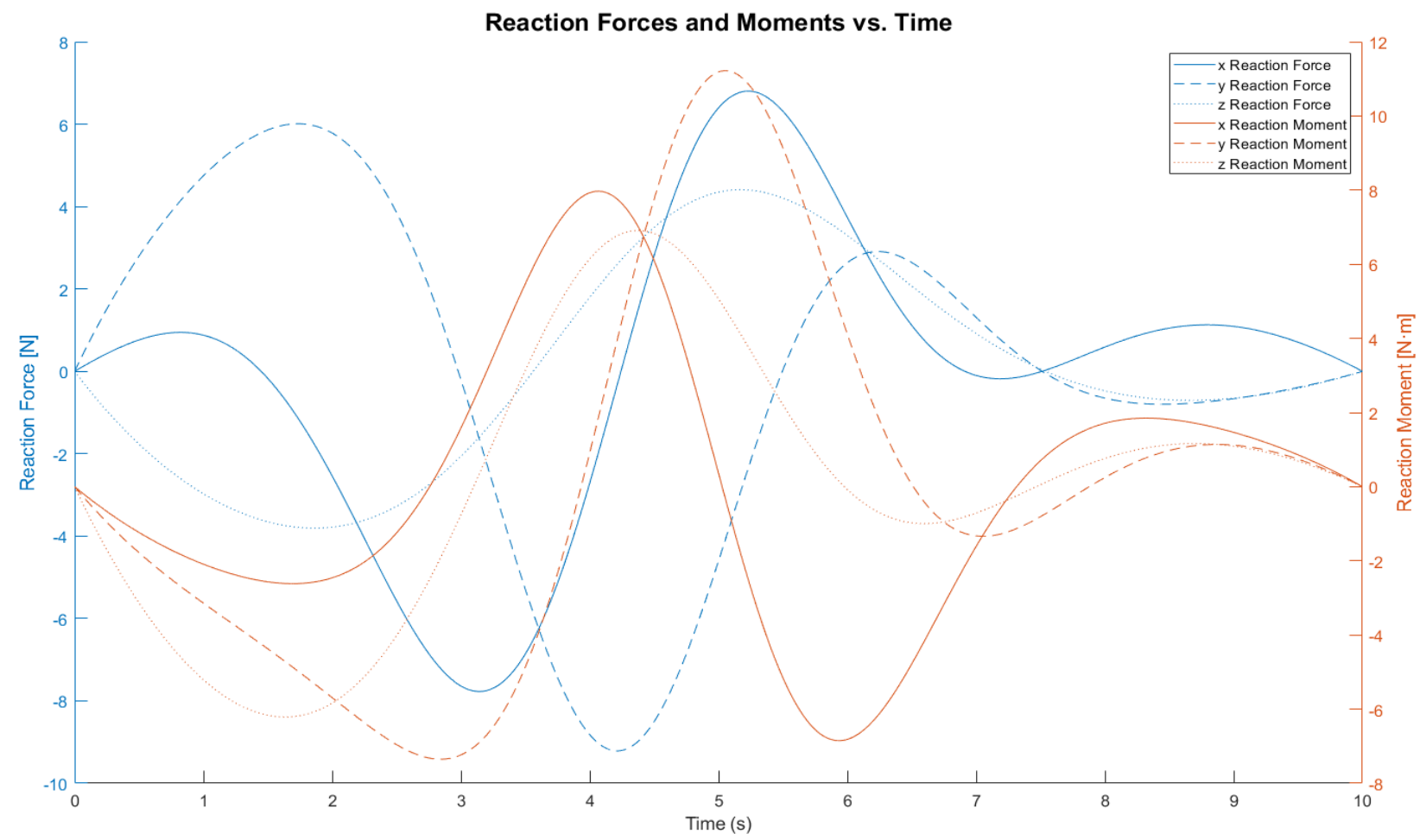

The forces and moments of the satellite disturbance load are plotted against time in FIGURE 5.

The objective of this work is to demonstrate the use of a PID controller with load estimative capacity in the control of position and attitude of satellites with onboard robotic manipulators. The simulation was run for multiple scenarios: without control, with non-predictive control, and with the load estimating controller. For runs with the controller, it is assumed that the satellite has an ideal control actuator onboard that can instantly induce the required torques or forces determined by the PID controller. It is important to note that this is a preliminary assumption used to evaluate the effectiveness of the control scheme rather than to evaluate its performance in a realistic application. The position and at- 
titude responses of the satellite both with and without control are compared in FIGURE 6. The left side of FIGURE 6 shows the uncontrolled case: notice that the angular and translational positions diverge from the reference (initial condition). The controlled case is shown on the right side of FIGURE 6. Notice how the satellite's deviation from the reference was drastically reduced compared to the uncontrolled case and that the satellite returned to the initial reference state (position of $(0,0,0)$ and angular position of $(0,0,0))$ after the disturbance loads ceased. It is important to note that this simulation represents a "worst case scenario" for the satellite, as several assumptions were made that negatively impact the control effectiveness. Despite these assumptions, the PID controller was very effective at stabilizing the system. Thus, we have succeeded in designing an effective controller for the satellite.
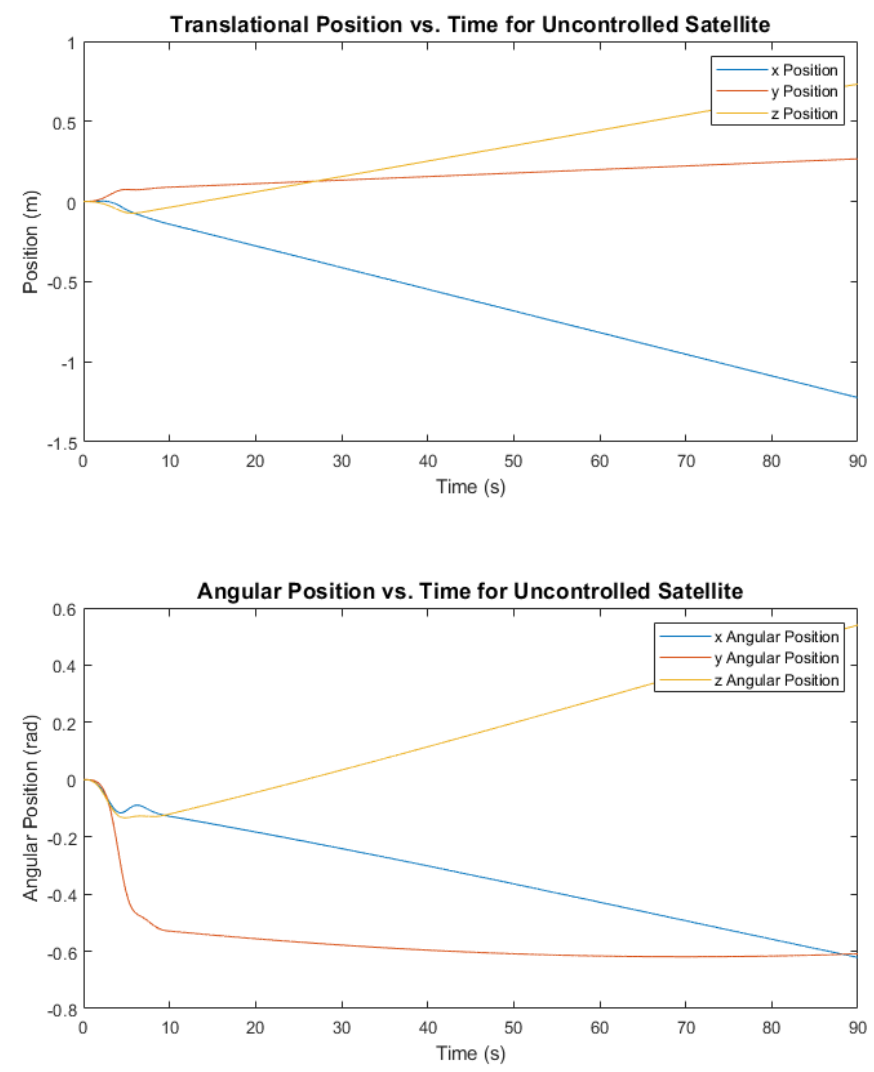

\section{Discussion Of SimULATION Results}

The use of the Proportional-Integral-Derivative control scheme with load prediction resulted in a very accurate satellite response. The maximum deviations from reference position and attitude were $.07 \mathrm{~mm}$ and $1.26^{\circ}$ respectively. Without the use of the controller, the satellite motion would continue indefinitely following a disturbance. These results provide evidence that such a control scheme could effectively maintain satellite position and attitude during robotic servicing operations. The use of calculated robotic arm disturbances as load predictions is a reasonable assumption when considering the actual implementation of robotic servicing missions. If a space organization were to implement satellitemounted robotic arms for maintenance purposes,
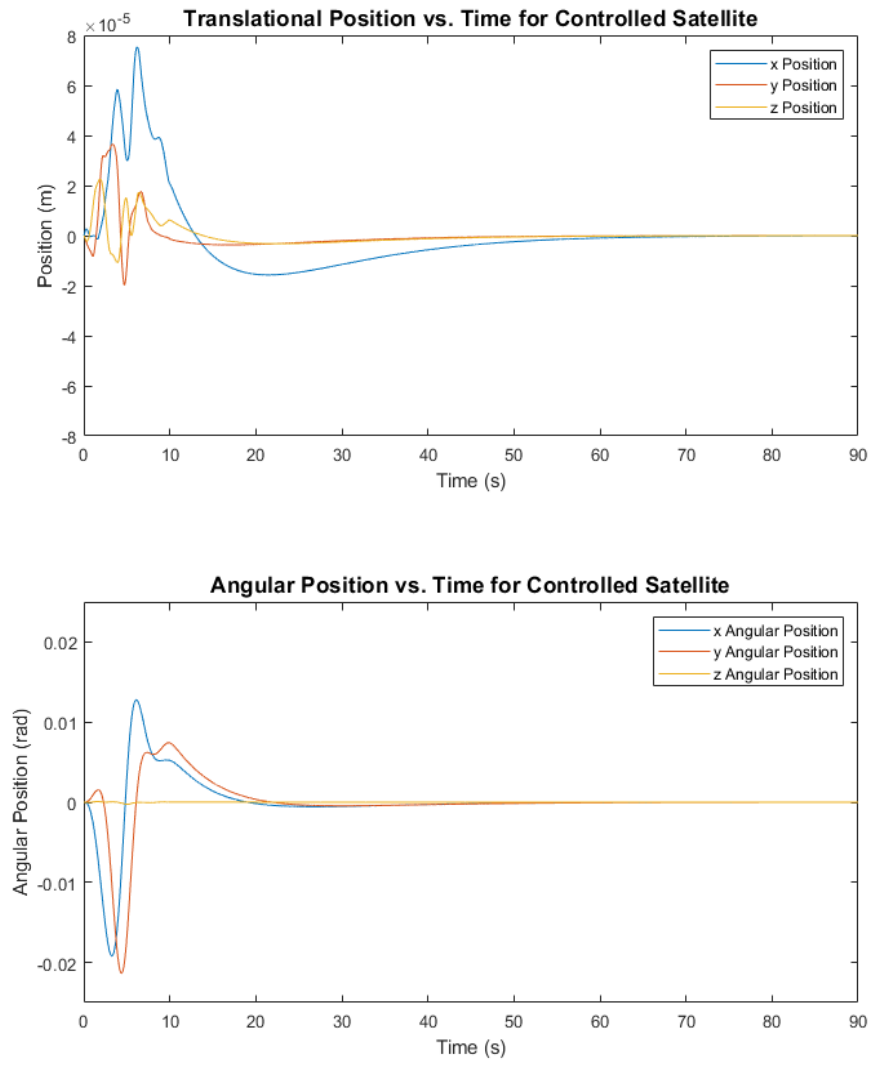

FIGURE 6: Comparison of system response between uncontrolled (LEFT) and controlled (RIGHT) satellite position (TOP) and angular position (ВОтTом) 
the simulation of the robot dynamics prior to the execution of the mission would result in a more accurately controlled system during operation, as the result of the simulation would be used as the load estimation. In addition to the load prediction, the PID controllers add robustness to the scheme, adjusting for additional perturbations arising from the operational environment.

\section{CONCLUSIONS AND FUTURE WORK}

The goal of this project was to create a dynamics simulation of a robotic manipulator and satellite in order to develop a model-based control algorithm for maintaining satellite position and orientation. Even with a very basic PID feedback control scheme, the results of the simulation were very promising. The arm load estimation technique allowed the controller to effectively eliminate translational and angular displacement. We believe that this research offers a framework for future investigation into more advanced control methods, including nonlinear approaches such at Lyapunov theory, which incorporates the nonlinear system model into the controls equation. We also hope to develop a more accurate satellite model that includes realistic actuators, such as reaction wheels and gas thrusters, to better evaluate the effectiveness of the control scheme. With this, we also hope to investigate various satellite/arm designs and to determine which configuration is most effective.

This was inspired by DAPRA's Robotic Servicing of Geosynchronous Satellites (RSGS) ${ }^{[4]}$ proposal, which aims to extend the operational lives of satellites located in geostationary orbit through issue diagnosis, repair, and upgrades. The implications of this proposal include lowering the cost of operation for geosynchronous satellites, thereby encouraging more private companies to contribute to space infrastructure. This, in turn, aligns closely with the NASA Space Technology Mission Directorate ${ }^{[18]}$, as contributing to this Earth-based infrastructure will facilitate future missions carried out to the moon any beyond. We hope future work on this project will incentivize more private companies to begin developing autonomous, robotic satellite systems to further humanities reach beyond planet Earth

\section{ACKNOWLEDGEMENTS}

The authors would like to acknowledge the help of others who contributed to the success of this project. We thank our Aresty research advisor, Dr. Annalisa Scacchioli, for her continuous guidance and encouragement throughout the duration of the project. We thank Dr. Aaron Mazzeo for introducing us to the robotics toolbox. We thank Dr. Laurent Burlion for his discussion about control methodologies and satellite attitude dynamics. We thank Mike Vivar for his recommendation of the robotics toolbox. Finally, we thank our undergraduate peers, especially Michael Higgins and Aaron Pfister, for their continued interest in our project.

\section{REFERENCES}

[1] F. Garcia, L. Peret, G. Verfaillie, Deployment and Maintenance of a Constellation of Satellites: a Benchmark, 2003.

[2] Defense Advanced Research Projects Agency. Robotic Servicing of Geosynchronous Satellites (RSGS).

HTTPS://WWW.DARPA.MIL/PROGRAM/ROBOTIC-SERVICING-OF-GEOSYNCHRONOUS-SATELLITES

[3] A. Antonello, P. Tsiotras, A. Valverde, Free-flying Spacecraftmounted Manipulators: A Tool for Simulating Dynamics and Control, 2019.

[4] Defense Advanced Research Projects Agency. (2019, May 22). Robotic Servicing of Geosynchronous Satellites Proposers Day.

[5] P.I. Corke, "Robotics, Vision \& Control", Springer 2017, ISBN 978-3-319-54413-7.

[6] MathWorks. 6DOF (Euler Angles). HTTPS://WWW.MATHWORKS.COM/HELP/AEROBLKS/6DOFEULERANGLES.HTML

[7] P.C. Hughes, "Spacecraft Attitude Dynamics", Dover Publications 2004, ISBN 0-486-43925-9

[8] Northrop Grumman. Space Logistics Services. HTTPS://WWW.NORTHROPGRUMMAN.COM/SPACE/SPACE-LOGISTICS-SER. VICES/

[9] Phipps, Claude \& Albrecht, G. \& Friedman, H. \& Gavel, D. \& George, E. \& Murray, J. \& Ho, Chunching \& Priedhorsky, W. \& Michaelis, M.M. \& Reilly, J.P.. (1996). ORION: Clearing near-Earth space debris using a 20-kW, 530-nm, Earth-based, repetitively pulsed laser. March 1996.

[10] Spectrolab. Space Solar Panels. HTTPS://WWW.SPECTROLAB.COM/DATASHEETS/PANEL/PANELS.PDF

[11] MathWorks. Open PID Tuner. HTTPS://WWW.MATHWORKS.COM/HELP/SLCONTROL/UG/DESIGNING-CONTROLLERS-WITH-THE-PID-TUNER.HTML

[12] G.F. Franklin, J.D. Powell, A. Emami-Naeini, "Feedback Control of Dynamic Systems", Pearson 2015, ISBN 978-0-13349659-8

[13] MathWorks. Sgolayfilt. HTTPS://WWW.MATHWORKS.COM/HELP/SIGNAL/REF/SGOLAYFILT.HTML 
[14] C.J. Damaren, A.H.J. de Ruiter, J.R. Forbes, "Spacecraft Dynamics and Control: An Introduction", Wiley 2013, ISBN 9781-118-34236-7

[15] S.A. Rawashdeh, Attitude Analysis of Small Satellites Using Model-Based Simulation, International Journal of Aerospace Engineering, 2019.
[16] R. Hernandez-Alvarado, L.G. Garcia-Valdovinos, et. al., Neural Network-Based Self-Tuning PID Control for Underwater Vehicles, Sensors, 2016.

[17] H.E.Soken, C. Hajiyev, S. Sakai, Robust Kalman filtering for small satellite attitude estimation in the presence of measurement faults, European Journal of Control, 2013.

[18] NASA. Space Technology Mission Directorate. HTTPS://WWW.NASA.GOV/DIRECTORATES/SPACETECH/ABOUT_US/INDEX.HTML 
8 SUPPLEMENTARY TABLES

\begin{tabular}{ccccc}
\hline JOINT & $d$ & $a$ & $\alpha$ & OFFSET \\
\hline 1 & 0.3469785 & 0 & $-\frac{\pi}{2}$ & 0 \\
\hline 2 & 0.2517295 & 0 & $-\frac{\pi}{2}$ & 0 \\
\hline 3 & 0.8776515 & 0 & $\frac{\pi}{2}$ & 0 \\
\hline 4 & 0.2122692 & 0 & $-\frac{\pi}{2}$ & 0 \\
\hline 5 & 0.1592019 & 0 & $\left(\frac{127}{449}\right)$ & 0 \\
\hline 5 A & 0.6349236746 & 0 & $-\left(\frac{127}{449}\right)$ & 0 \\
\hline $5 B$ & 0.1074953 & 0 & $\frac{\pi}{2}$ & 0 \\
\hline 6 & 0.1728089 & 0 & $-\frac{\pi}{2}$ & 0 \\
\hline 7 & 0.2000229 & 0 & 0 & 0 \\
\hline
\end{tabular}

SUPPLEMENTAL TABLE S1: Denavit-Hartenberg parameters for the FREND Mark II arm model.

\begin{tabular}{cccc}
\hline LINK & $m(\mathrm{~kg})$ & $r(m)$ & $L(m)$ \\
\hline 1 & 2.45581930238976 & 0.0530673 & 0.3469785 \\
\hline 2 & 1.78167282722394 & 0.0530673 & 0.2517295 \\
\hline 3 & 8.64176380422475 & 0.0625922 & 0.8776515 \\
\hline 4 & 0.8023083874777559 & 0.03877995 & 0.2122692 \\
\hline 5 & 0.540058000435033 & 0.0367389 & 0.1592019 \\
\hline $5 \mathrm{~A}$ & 2.15384119253858 & 0.0367389 & 0.6349236746 \\
\hline $5 \mathrm{~B}$ & 0.364654547302287 & 0.0367389 & 0.1074953 \\
\hline 6 & 0.425389148502536 & 0.0312961 & 0.1728089 \\
\hline 7 & 0.536125954214708 & 0.0326568 & 0.2000229 \\
\hline
\end{tabular}

SUPPLEMENTAL TABLE S2: Mass, radius, and length of each cylindrical link object used in the definition of the FREND arm model 


\begin{tabular}{|c|c|}
\hline LINK & $I\left(k g \cdot m^{2}\right)$ \\
\hline 1 & $(0.007780417725678780000 .01167062658851820000 .00778041772567878)$ \\
\hline 2 & $(0.00564461678137480000 .00846692517206220000 .0056446167813748)$ \\
\hline 3 & $(0.03808862960664150000 .05713294440996230000 .0380886296066415)$ \\
\hline 4 & $(0.001357401561525320000 .002036102342287980000 .00135740156152532)$ \\
\hline 5 & 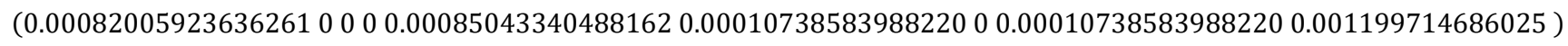 \\
\hline $5 \mathrm{~A}$ & $\begin{array}{c}(0.0032705327245900000 .003391669964095-0.000428272602659950 \\
-0.000428272602659950 .004784661847380)\end{array}$ \\
\hline $5 B$ & $(0.0005537152108773180000 .0008305728163159760000 .000553715210877318)$ \\
\hline 6 & $(0.0004687263527173910000 .0007030895290760870000 .000468726352717391)$ \\
\hline 7 & $(0.0006432304682092750000 .0006432304682092750000 .000964845702313913)$ \\
\hline
\end{tabular}

SUPPLEMENTAL TABLE S3: Inertia tensors for each link of the FREND Mk. // arm model

\begin{tabular}{cccc}
\hline & $K_{P}$ & $K_{I}$ & $K_{D}$ \\
\hline FORCE & 617.061827129926 & 35.6866537699081 & 2667.42085820047 \\
\hline MOMENT & 102.843637854979 & 5.94777562634487 & 444.570143033412 \\
\hline
\end{tabular}

SUPPLEMENTAL TABLE S3: Proportional, integral, and derivative gains for the force and moment PID controllers 